\title{
A Triangle Algorithm of Padé-Type Approximant for Two-Dimensional Fredholm Integral Equations of the Second Kind
}

\author{
Meilan Sun $\mathbb{D}^{1,2}$ and Chuanqing Gu ${ }^{1}{ }^{1}$ \\ ${ }^{1}$ Department of Mathematics, Shanghai University, Shanghai 200444, China \\ ${ }^{2}$ Department of Mathematics and Physics, HeFei University, HeFei 230601, China \\ Correspondence should be addressed to Chuanqing Gu; cqgu@staff.shu.edu.cn
}

Received 26 March 2018; Revised 29 May 2018; Accepted 16 July 2018; Published 25 July 2018

Academic Editor: Kishin Sadarangani

Copyright (C) 2018 Meilan Sun and Chuanqing Gu. This is an open access article distributed under the Creative Commons Attribution License, which permits unrestricted use, distribution, and reproduction in any medium, provided the original work is properly cited.

\begin{abstract}
The function-valued Padé-type approximation (2DFPTA) is used to solve two-dimensional Fredholm integral equation of the second kind. In order to compute 2DFPTA, a triangle recursive algorithm based on Sylvester identity is proposed. The advantage of this algorithm is that, in the process of calculating 2DFPTA to avoid the calculation of the determinant, it can start from the initial value, from low to high order, and gradually proceeds. Compared with the original two methods, the numerical examples show that the algorithm is effective.
\end{abstract}

\section{Introduction}

Consider a two-dimensional Fredholm integral equation of the second kind (2DF-II):

$$
f(x, \lambda)=g(x)+\lambda \int_{D} K(x, y) f(y, \lambda) d y
$$

$$
x, y \in D \text {, }
$$

where $D$ is a bounded domain, $x=\left(x_{1}, x_{2}\right), y=\left(y_{1}\right.$, $\left.y_{2}\right), d y=d y_{1} d y_{2}, K$ and $f$ are given functions defined on $D$, and $\lambda$ is a real parameter called a characteristic value. It is known that a sequence of approximations leading to an infinite power series can be yielded by successive substitution, which is an iterative procedure, from this kind of integral equation. Set $f(x, \lambda)$ to be analytic and meromorphic in a neighborhood $\widetilde{D}$ of the origin $\lambda=0$. Let its Neumann series be given by

$$
f(x, \lambda)=\sum_{i=0}^{\infty} c_{i}(x) \lambda^{i}
$$

in which $c_{i}(x)$ are continuous on $D$ and $c_{0}(x)=g(x)$,

$$
c_{i}(x)=\int_{D} K(x, y) c_{i-1}(y) d y, \quad \text { for } i=1,2, \ldots
$$

Let $c_{i}(x), c_{j}(x) \in L^{2}[a, b]$ and the inner product and the norm be defined by

$$
\begin{aligned}
\left(c_{i}, c_{j}\right) & =\int_{D} c_{i}(x) c_{j}(x) d x, \\
\left\|c_{i}\right\| & =\left(c_{i}, c_{i}\right)^{1 / 2}=\left(\int_{D} c_{i}^{2}(x) d x\right)^{1 / 2} .
\end{aligned}
$$

Two-dimensional Fredholm integral equation of the second kind (2DF-II) is a useful tool to model many problems arising in fracture mechanics, transportation [1], 2D electromagnetic scattering [2], and computer graphics manipulations [3]. For domain $D$, some works treat the square case [4-10] and some are based on discrete Galerkin method [5], Monte Carlo methods [6], or piecewise approximating polynomials [7], Nytröm methods based on cubature rules obtained as the tensor product of two-variate Gaussian 
rules $[8,10]$, and meshless method with complex factors [11]. Rational approximation has extensive application in engineering, technology and calculation [12-18]. As efficient rational approximating techniques, a generalized inverse function-valued Padé approximants had been used in solving integral equation $[13,14]$, and a function-valued Padé-type approximation method was used to solve one and twodimensional Fredholm integral equation of the second kind [15-18].

In the paper, the function-valued Padé-type approximation (2DFPTA) is used to solve 2DF-II. In order to compute 2DFPTA, a recursive algorithm based on Sylvester identity is proposed. The remainder of this paper is organized as follows. In Section 2, we give the definition of 2DFPTA [18]. In Section 3, we apply Sylvester identity to propose a recursive algorithm to compute 2DFPTA. In Section 4, we compare our algorithm with the two algorithms in $[4,18]$.

\section{Function-Valued Padé-Type Approximants and Its Convergence Analysis}

In this section, we will give the definition of the functionvalued Padé-type approximation [15-18]. Let $\phi^{(l)}: \mathbf{P} \longrightarrow \mathbb{C}$ be a linear functional on the polynomial space $\mathbf{P}$, and define it by

$$
\phi^{(l)}\left(t^{i}\right)=c_{l+i}\left(x_{1}, x_{2}\right), \quad i=0,1, \ldots, l \in \mathbb{Z},
$$

where $\phi^{(l)}\left(t^{i}\right)=0$ for $l+i<0$. We can obtain from linear functional $\phi=\phi^{(0)}$ in (6) that

$$
\begin{aligned}
\phi\left((1-t \lambda)^{-1}\right) & =\phi\left(1+t \lambda+(t \lambda)^{2}+\cdots\right) \\
& =\sum_{i=0}^{\infty} c_{i}\left(x_{1}, x_{2}\right) \lambda^{i}=f(x, \lambda) .
\end{aligned}
$$

Let

$$
v_{n}(\lambda)=b_{0}+b_{1} \lambda+\cdots+b_{n} \lambda^{n}
$$

where $v_{n} \in \mathbf{P}_{n}$ is a scalar polynomial of degree $n$ and assume $b_{n} \neq 0$. by

Define the polynomial $w$ with function-valued coefficient

$$
w_{m}(x, \lambda)=\phi\left(\frac{t^{m-n+1} v_{n}(t)-\lambda^{m-n+1} v_{n}(\lambda)}{t-\lambda}\right) .
$$

Note that $\phi$ acts on $P$ and $w_{m}(x, \lambda)$ is a function-valued polynomial in $\lambda$ of degree $m$.

Set

$$
\begin{aligned}
\widetilde{v}_{n}(\lambda) & =\lambda^{n} v_{n}\left(\lambda^{-1}\right)=\sum_{j=0}^{n} b_{j} \lambda^{n-j} . \\
\widetilde{w}_{m}(x, \lambda) & =\lambda^{m} w_{m}\left(x, \lambda^{-1}\right)
\end{aligned}
$$

The polynomial $v_{n}$ is called the generating polynomial of the Padé-type approximation $(m / n)$. It can be arbitrarily chosen and we have $n$ degrees of freedom.
It is known from $(10)$ that $b_{n} \neq 0$ implies $\widetilde{v}_{n}(0) \neq 0$. In view of (8), (9), and (11), $\widetilde{w}_{m}(x, \lambda)$ is of the following form:

$$
\widetilde{w}_{m}(x, \lambda)=\sum_{j=0}^{n} b_{j} \lambda^{n-j} \sum_{i=0}^{m-n+j} c_{i}(x) \lambda^{i}=\sum_{i=0}^{m} \sum_{j=0}^{n} b_{j} \tilde{c}_{i}^{j}(x) \lambda^{i},
$$

in which $\widetilde{c}_{i}^{j}(x)=c_{i-n+j}(x)$. Using (10) and (12), we get

$$
\begin{aligned}
\widetilde{v}_{n}(\lambda) f(x, \lambda)-\widetilde{w}_{m}(x, \lambda) & \left(\sum_{j=0}^{n} b_{j} \lambda^{n-j}\right)\left(\sum_{i=0}^{\infty} c_{i}(x) \lambda^{i}\right) \\
& -\sum_{j=0}^{n} b_{j} \lambda^{n-j} \sum_{i=0}^{m-n+j} c_{i}(x) \lambda^{i} . \\
= & \sum_{i=0}^{\infty}\left(\sum_{j=0}^{n} c_{m-n+1+i+j}(x)\right) \lambda^{m+1+i} \\
= & \sum_{i=m+1}^{\infty}\left(\sum_{j=0}^{n} b_{j} \widetilde{c}_{i}^{j}(x)\right) \lambda^{i}=O\left(\lambda^{m+1}\right) .
\end{aligned}
$$

Definition 1. Let $\widetilde{w}_{m}(x, \lambda)$ and $\widetilde{v}_{n}(\lambda)$ be given by (12) and (10), respectively, and then the rational function

$$
\begin{aligned}
R_{m, n}(x, \lambda) & =\frac{\widetilde{w}_{m}(x, \lambda)}{\widetilde{v}_{n}(\lambda)} \\
& =\frac{\sum_{j=0}^{n} b_{j} \lambda^{n-j} \sum_{i=0}^{m-n+j} c_{i}(x) \lambda^{i}}{\sum_{j=0}^{n} b_{j} \lambda^{n-j}} \\
& =\frac{\sum_{i=0}^{m} \sum_{j=0}^{n} b_{j} \tilde{c}_{i}^{j}(x) \lambda^{i}}{\sum_{j=0}^{n} b_{j} \lambda^{n-j}}
\end{aligned}
$$

is defined as a two-dimensional function-valued Padé-type approximation (2DFPTA) with generating polynomial $v_{n}(\lambda)$ and is denoted by $(m / n)_{f}(x, \lambda)$.

According to the relations (9) and (11), we can from [19] obtain the following error formula with the linear functional form:

$$
\begin{aligned}
f(x, \lambda)-\left(\frac{m}{n}\right)_{f}(x, \lambda)=\frac{\lambda^{m+1}}{\widetilde{v_{n}}(\lambda)} \phi^{(m-n+1)}\left(\frac{v_{n}(t)}{1-t \lambda}\right) \\
=\frac{\lambda^{m+1}}{\widetilde{v}_{n}(\lambda)} \phi^{(m-n+1)}\left[v_{n}(t)\right. \\
\left.\cdot\left(1+t \lambda+\cdots+t^{n-1} \lambda^{n-1}+\frac{t^{n} \lambda^{n}}{1-t \lambda}\right)\right] .
\end{aligned}
$$

Note that the error formula (15) is disadvantageous to estimate error numerically, and it implies from (13) that the Padé-type approximation property is

$$
f(x, \lambda)-\left(\frac{m}{n}\right)_{f}(x, \lambda)=O\left(\lambda^{m+1}\right)
$$


In order to compute conveniently, we choose $\max _{x \in D}\left|\sum_{j=0}^{n} b_{j} \tilde{c}_{i}^{j}(x)\right|$ as the maximum absolute value of the coefficient of $\lambda^{m+1}$ on $D$ in (13). We can also observe from (15) and (16) that if the coefficients are given, the order of the approximation is $\lambda^{m+1}$.

\section{A Triangle Recursive Algorithm$$
\text { of }(m / n)_{f}(x, \lambda)
$$

As the definition given in Section 2, we find that the key to calculating 2DFPTA is to compute its the generating polynomial $v_{n}(\lambda)$. If the generator polynomial $v_{n}(\lambda)$ is determined, we can compute the 2DFPTA of type $(m, n)$ according to (10) and (12). In the section, we will apply Sylvester's identity [20] to propose a three-term recurrence formula for computing $\widetilde{v}_{n}(\lambda)$ and establish a complete recursive algorithm for calculating 2DFPTA.

Lemma 2 (Sylvester identity [20]). Let

$$
\begin{aligned}
A & =\left(\begin{array}{cccc}
a_{0,1} & a_{0,2} & \cdots & a_{0, n} \\
a_{1,1} & a_{1,2} & \cdots & a_{1, n} \\
\vdots & \vdots & \ddots & \vdots \\
a_{n-1,1} & a_{n-1,2} & \cdots & a_{n-1, n}
\end{array}\right), \\
B & =\left(\begin{array}{cccc}
a_{0,1} & a_{0,2} & \cdots & a_{0, n-1} \\
a_{1,1} & a_{1,2} & \cdots & a_{1, n-1} \\
\vdots & \vdots & \ddots & \vdots \\
a_{n-2,1} & a_{n-2,2} & \cdots & a_{n-2, n-1}
\end{array}\right),
\end{aligned}
$$

and then it holds that

$$
\left|\begin{array}{ll}
x_{0} & x \\
u & A
\end{array}\right||B|=|A|\left|\begin{array}{cc}
x_{0} & x^{\prime} \\
u^{\prime} & B
\end{array}\right|-\left|A^{\prime}\right|\left|\begin{array}{cc}
x^{\prime} & x_{n} \\
B & u^{\prime \prime}
\end{array}\right|,
$$

where $A^{\prime}=\left(\begin{array}{cc}u^{\prime} & B \\ a_{n-1,0} & w\end{array}\right)$ and

$$
\begin{aligned}
x & =\left(x_{1}, x_{2}, \ldots, x_{n}\right), \\
x^{\prime} & =\left(x_{1}, x_{2}, \ldots, x_{n-1}\right), \\
u & =\left(a_{0,0}, a_{1,0}, \ldots, a_{n-1,0}\right)^{T}, \\
u^{\prime} & =\left(a_{0,0}, a_{1,0}, \ldots, a_{n-2,0}\right), \\
u^{\prime \prime} & =\left(a_{0, n}, a_{1, n}, \ldots, a_{n-2, n}\right)^{T}, \\
w & =\left(a_{n-1,1}, a_{n-1,2}, \ldots, a_{n-1, n-1}\right) .
\end{aligned}
$$

Set $b_{0}=1$, the generating polynomial $v_{n}(\lambda)=b_{0}+b_{1} \lambda+\cdots+$ $b_{n} \lambda^{n}$.
Lemma 3 (see [15]). Let the determinant $\left|A_{n}\right| \neq 0$; then the generating polynomial $v_{n}(\lambda)$ exists and is unique and can be expressed as

$$
v_{n}(\lambda)=\frac{\left|\begin{array}{cc}
\vec{\eta}^{(n)} & \vec{\lambda}^{(n)} \\
\vec{A}_{n}
\end{array}\right|}{\left|A_{n}\right|},
$$

where

$$
\begin{aligned}
& A_{n} \\
& =\left(\begin{array}{cccc}
\left(c_{m-n+1}, c_{m-n+2}\right) & \left(c_{m-n+1}, c_{m-n+3}\right) & \ldots & \left(c_{m-n+1}, c_{m+1}\right) \\
\left(c_{m-n+1}, c_{m-n+3}\right) & \left(c_{m-n+1}, c_{m-n+4}\right) & \ldots & \left(c_{m-n+1}, c_{m+2}\right) \\
\vdots & \vdots & \ddots & \vdots \\
\left(c_{m-n+1}, c_{m+1}\right) & \left(c_{m-n+1}, c_{m+2}\right) & \ldots & \left(c_{m-n+1}, c_{m+n}\right)
\end{array}\right), \\
& \vec{\lambda}^{(n)}=\left(\lambda, \lambda^{2}, \ldots, \lambda^{n}\right), \\
& \vec{\eta}^{(n)}=\left(\left(c_{m-n+1}, c_{m-n+1}\right),\left(c_{m-n+1}, c_{m-n+2}\right), \ldots,\left(c_{m-n+1}, c_{m}\right)\right)^{T} .
\end{aligned}
$$

Definition 4. For any $j$ th degree polynomial $v_{j}(\lambda)=b_{0}+b_{1} \lambda+$ $\cdots+b_{j} \lambda^{j}$, by the higher-order linear functional $\phi^{(l)}\left(\lambda^{j}\right)=c_{l+j}$, we define the vector inner product with $l$ order as follows:

$$
\begin{aligned}
{\left[c_{l}, v_{j}(\lambda)\right]_{l}=} & \left(c_{l}, \phi^{(l)}\left(v_{j}(\lambda)\right)\right) \\
= & \left(c_{l}, \phi^{(l)}\left(b_{0}+b_{1} \lambda+\cdots+b_{j} \lambda^{j}\right)\right) \\
= & \left(c_{l}, b_{0} c_{l}+b_{1} c_{l+1}+\cdots+b_{j} c_{l+j}\right) \\
= & b_{0}\left(c_{l}, c_{l}\right)+b_{1}\left(c_{l}, c_{l+1}\right)+\cdots \\
& +b_{j}\left(c_{l}, c_{l+j}\right),
\end{aligned}
$$

where $l=m-n+1, j=0,1,2, \ldots$.

To discuss conveniently, we introduce the following notations:

$$
\begin{gathered}
G_{k}^{(i)}=\left|\begin{array}{cccc}
\lambda^{i} & \lambda^{i+1} & \cdots & \lambda^{i+k} \\
{\left[c_{l}, \lambda^{i}\right]_{l}} & {\left[c_{l}, \lambda^{i+1}\right]_{l}} & \cdots & {\left[c_{l}, \lambda^{i+k}\right]_{l}} \\
\vdots & \vdots & \cdots & \vdots \\
{\left[c_{l}, \lambda^{i+k-1}\right]_{l}} & {\left[c_{l}, \lambda^{i+k}\right]_{l}} & \cdots & {\left[c_{l}, \lambda^{i+2 k-1}\right]_{l}}
\end{array}\right|, \\
D_{k}^{(i)}=\left|\begin{array}{cccc}
{\left[c_{l}, \lambda^{i+1}\right]_{l}} & {\left[c_{l}, \lambda^{i+2}\right]_{l}} & \cdots & {\left[c_{l}, \lambda^{i+k}\right]_{l}} \\
{\left[c_{l}, \lambda^{i+2}\right]_{l}} & {\left[c_{l}, \lambda^{i+3}\right]_{l}} & \cdots & {\left[\mathcal{c}_{l}, \lambda^{i+k+1}\right]_{l}} \\
\vdots & \vdots & \cdots & \vdots \\
{\left[c_{l}, \lambda^{i+k}\right]_{l}} & {\left[c_{l}, \lambda^{i+k+1}\right]_{l}} & \cdots & {\left[c_{l}, \lambda^{i+2 k-1}\right]_{l}}
\end{array}\right| .
\end{gathered}
$$

Let

$$
v_{k}^{(i)}=\frac{G_{k}^{(i)}}{D_{k}^{(i)}},
$$


where if $i=0, k=n$ and

$$
\begin{aligned}
& G_{n}^{(0)}=\left|\begin{array}{cc}
1 & \vec{\lambda}^{(n)} \\
\vec{\eta}^{(n)} & A_{n}
\end{array}\right|, \\
& D_{n}^{(0)}=\left|A_{n}\right| .
\end{aligned}
$$

From (20) and (25), we conclude that

$$
v_{n}^{(0)}=v_{n}(\lambda)
$$

To compute $v_{n}^{(0)}$, we give the following theorem. According to Definition 4 and the $j$ th degree polynomial $v_{k-1}^{(i)}(\lambda)=$ $b_{0}+b_{1} \lambda+\cdots+b_{j} \lambda^{j}$, we gain the vector inner product with $l+k-1$ order as follows:

$$
\begin{aligned}
{\left[\mathcal{c}_{l}, v_{k-1}^{(i)}(\lambda)\right]_{l+k-1}=} & \left(c_{l}, \phi^{(l+k-1)}\left(v_{k-1}^{(i)}(\lambda)\right)\right) \\
= & b_{0}\left(c_{l}, c_{l+k-1}\right)+b_{1}\left(c_{l}, c_{l+k}\right)+\cdots \\
& +b_{j}\left(c_{l}, c_{l+k-1+j}\right),
\end{aligned}
$$

where $l=m-n+1, k=1,2, \ldots, n, i=j-(k-1)$.

Theorem 5 (three-term recurrence formula). Let $D_{k}^{(i)} D_{k-1}^{(i)} \neq$ 0 for all $k=1,2, \ldots, n, i=0,1,2, \ldots, n$. Then it holds that

$$
v_{k}^{(i)}=v_{k-1}^{(i)}-\frac{\left(c_{l}, \phi^{(l+k-1)}\left(v_{k-1}^{(i)}\right)\right)}{\left(c_{l}, \phi^{(l+k-1)}\left(v_{k-1}^{(i+1)}\right)\right)} v_{k-1}^{(i+1)},
$$

where the initial value is $v_{0}^{(i)}=\lambda^{i}$.

Proof. Set $x_{0}=\lambda^{i}, x=\left(\lambda^{i+1}, \lambda^{i+2}, \ldots, \lambda^{i+k}\right), u=\left(\left[c_{1}, \lambda^{i}\right]_{1}\right.$, $\left.\left[c_{2}, \lambda^{i}\right]_{2}, \ldots,\left[c_{k}, \lambda^{i}\right]_{k}\right)$ and $|A|=D_{k}^{(i)},|B|=D_{k-1}^{(i)}$. Substitute them into the formula (18) and we conclude that

$$
G_{k}^{(i)} D_{k-1}^{(i)}=G_{k-1}^{(i)} D_{k}^{(i)}-G_{k-1}^{(i+1)} D_{k}^{(i-1)} .
$$

Due to $D_{k}^{(i)} D_{k-1}^{(i)} \neq 0$, we have

$$
\frac{G_{k}^{(i)}}{D_{k}^{(i)}}=\frac{G_{k-1}^{(i)}}{D_{k-1}^{(i)}}-\frac{G_{k-1}^{(i+1)} D_{k}^{(i-1)}}{D_{k-1}^{(i)} D_{k}^{(i)}} .
$$

By (25) we get $G_{k-1}^{(i+1)}=D_{k-1}^{(i+1)} v_{k-1}^{(i+1)}$ and substitute it into the above formula; then

$$
\frac{G_{k}^{(i)}}{D_{k}^{(i)}}=\frac{G_{k-1}^{(i)}}{D_{k-1}^{(i)}}-\frac{D_{k}^{(i-1)} D_{k-1}^{(i+1)} v_{k-1}^{(i+1)}}{D_{k-1}^{(i)} D_{k}^{(i)}},
$$

that is,

$$
v_{k}^{(i)}=v_{k-1}^{(i)}-\frac{D_{k}^{(i-1)} D_{k-1}^{(i+1)}}{D_{k-1}^{(i)} D_{k}^{(i)}} v_{k-1}^{(i+1)} .
$$

According to the higher-order linear functional and nature of the determinant, we have

$$
\left(c_{l}, \phi^{(l+k-1)}\left(v_{k-1}^{(i)}\right)\right)=\frac{(-1)^{k-1} D_{k}^{(i-1)}}{D_{k-1}^{(i)}},
$$

and

$$
\left(c_{l}, \phi^{(l+k-1)}\left(v_{k-1}^{(i+1)}\right)\right)=\frac{(-1)^{k-1} D_{k}^{(i)}}{D_{k-1}^{(i+1)}} .
$$

Dividing (34) by (35), we deduce that

$$
\frac{\left(c_{l}, \phi^{(l+k-1)}\left(v_{k-1}^{(i)}\right)\right)}{\left(c_{l}, \phi^{(l+k-1)}\left(v_{k-1}^{i+1}\right)\right)}=\frac{D_{k}^{(i-1)} D_{k-1}^{(i+1)}}{D_{k}^{(i)} D_{k-1}^{(i)}} .
$$

From (33) and (36), we obtain that

$$
v_{k}^{(i)}=v_{k-1}^{(i)}-\frac{\left(c_{l}, \phi^{(l+k-1)}\left(v_{k-1}^{(i)}\right)\right)}{\left(c_{l}, \phi^{(l+k-1)}\left(v_{k-1}^{(i+1)}\right)\right)} v_{k-1}^{(i+1)} .
$$

Example 6. Let us choose an example of $n=6$ to illustrate the process to generate $v_{6}(\lambda)$. By the three-term recurrence relation (16), the calculation program of $v_{6}(\lambda)$ can be arranged according to the following bottom-down triangle:

$$
\begin{aligned}
& v_{0}^{(0)} \\
& \searrow \\
& v_{0}^{(1)} \longrightarrow v_{1}^{(0)} \\
& v_{0}^{(2)} \longrightarrow v_{1}^{(1)} \longrightarrow v_{2}^{(0)} \\
& \searrow \quad \searrow \quad \searrow \\
& v_{0}^{(3)} \longrightarrow v_{1}^{(2)} \longrightarrow v_{2}^{(1)} \longrightarrow v_{3}^{(0)} \\
& \searrow \quad \searrow \quad \searrow \quad \searrow \\
& v_{0}^{(4)} \longrightarrow v_{1}^{(3)} \longrightarrow v_{2}^{(2)} \longrightarrow v_{3}^{(1)} \longrightarrow v_{4}^{(0)} \\
& v_{0}^{(5)} \rightarrow v_{1}^{(4)} \rightarrow v_{2}^{(3)} \rightarrow v_{3}^{(2)} \rightarrow v_{4}^{(1)} \longrightarrow v_{5}^{(0)}
\end{aligned}
$$

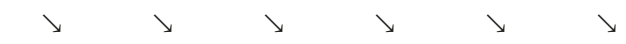

$$
\begin{aligned}
& v_{0}^{(6)} \longrightarrow v_{1}^{(5)} \longrightarrow v_{2}^{(4)} \longrightarrow v_{3}^{(3)} \longrightarrow v_{4}^{(2)} \longrightarrow v_{5}^{(1)} \longrightarrow v_{6}^{(0)}
\end{aligned}
$$

When computing $v_{k}^{(i)}$ we mainly use the left polynomial $v_{k-1}^{(i+1)}$, the polynomial $v_{k-1}^{(i)}$ in the top left corner in the table, and their relation (29). It is not difficult to find that, in every column, the number of polynomial is in decline when $\mathrm{k}$ is bigger and bigger.

It is noticed that $v_{k}^{(i)}$ can also be arranged by another pattern as follows: 


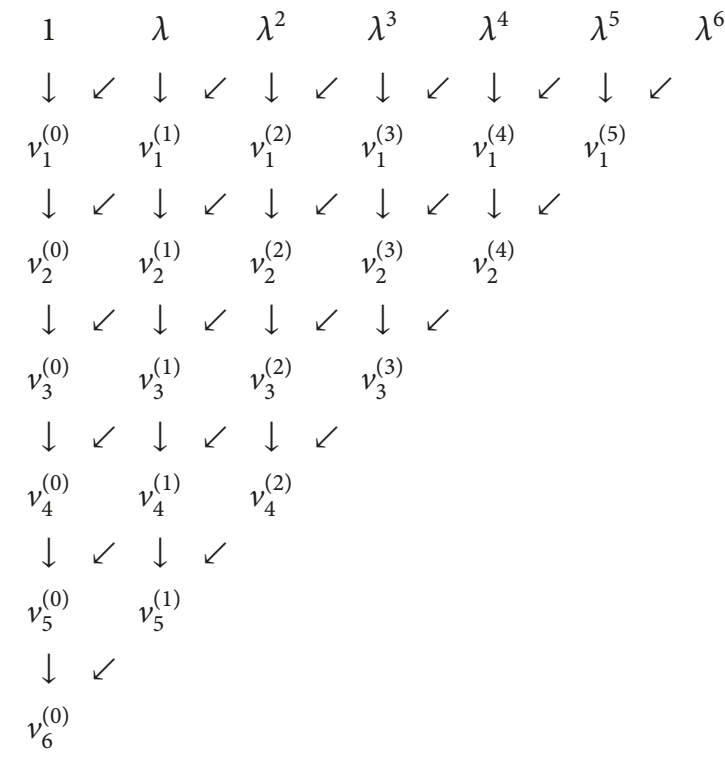

When computing $v_{k}^{(i)}$ we mainly use the above polynomial $v_{k-1}^{(i)}$, the polynomial $v_{k-1}^{(i+1)}$ in the top-right corner in the table, and their relation (29). Similarly, the number of polynomials is in decline when $k$ is bigger and bigger in every row. In this way, we can recursively get $v_{6}(\lambda)=v_{6}^{(0)}$.

From the three-term recurrence relation (29), we now build a complete recursive algorithm for calculating 2DFPTA. The advantage of this algorithm is that in the process of calculating 2DFPTA to avoid the calculation of the determinant, it can start from the initial value, from low to high order, and is gradually calculated. And its specific computational steps are as follows.

Algorithm 7 (Sylvester-type triangle algorithm).

(1) Set the initial value as $v_{0}^{(i)}=\lambda^{i}, i=0,1, \ldots, n$ compute $\left(c_{i}, c_{j}\right)$ by $(4)$.

(2) For $k=1,2, \ldots, n-1$ compute by (29) in turn $v_{k}^{(0)}$, $v_{k}^{(1)}, \ldots, v_{k}^{(n-k)}$.

(3) Compute $v_{n}(\lambda)=v_{n}^{(0)}=v_{n-1}^{(0)}-\left(\left(c_{l}, \phi^{(l+k-1)}\left(v_{n-1}^{(0)}\right)\right) /\left(c_{l}\right.\right.$, $\left.\left.\phi^{(l+k-1)}\left(v_{n-1}^{(1)}\right)\right)\right) v_{n-1}^{(1)}$.

(4) $v_{n}(\lambda)$ is written as $v_{n}(\lambda)=b_{0}+b_{1} \lambda+\cdots+b_{n} \lambda^{n}\left(b_{n}=1\right)$ and the denominator polynomial of function-valued Padé-type approximation is

$$
\widetilde{v}_{n}(\lambda)=\lambda^{n} v_{n}\left(\lambda^{-1}\right)=\sum_{i=0}^{n} b_{i} \lambda^{n-i}
$$

(5) From (12), numerator polynomial of $(m / n)_{f}(x, \lambda)$ is

$\widetilde{w}_{m}(x, \lambda)=\sum_{j=0}^{n} b_{j} \lambda^{n-j} \sum_{i=0}^{m-n+j} c_{i}(x) \lambda^{i}=\sum_{i=0}^{m} \sum_{j=0}^{n} b_{j} \tilde{c}_{i}^{j}(x) \lambda^{i}$.

(6) In view of Definition 1 , there exists $(m / n)_{f}(x, \lambda)$ such that

$$
\left(\frac{m}{n}\right)_{f}(x, \lambda)=\frac{\widetilde{w}_{m}(x, \lambda)}{\widetilde{v}_{n}(\lambda)}
$$

Example 8. Consider the second Fredholm integral equation

$$
\begin{gathered}
f\left(x_{1}, x_{2}, \lambda\right)=x_{1} \cos \left(x_{2}\right)+\frac{4}{3} \sin \left(x_{1}\right)-\left(1+\frac{4}{3}\right. \\
\cdot \sin (1)) x_{2}+\lambda \int_{-1}^{1} \int_{-1}^{1}\left(y_{2} \sin \left(x_{1}\right)+x_{2} y_{1}\right) \\
\cdot f\left(y_{1}, y_{2}, \lambda\right) d y_{1} d y_{2}, \quad x_{1}, x_{2} \in[-1,1] .
\end{gathered}
$$

Its kernel is $K(x, t)=\left(y_{2} \sin \left(x_{1}\right)+x_{2} y_{1}\right)$.

In the following we use the triangle algorithm to yield $(2 / 2)_{f}(x, \lambda)$ of the integral equation.

Solution. The first few terms of the power series for $f(x, \lambda)$ are given by

$$
\begin{aligned}
& f(x, \lambda)=c_{0}(x)+c_{1}(x) \lambda+c_{2}(x) \lambda^{2}+c_{3}(x) \lambda^{3} \\
& +c_{4}(x) \lambda^{4}+\cdots=x_{1} \cos \left(x_{2}\right)+\frac{4}{3} \sin \left(x_{1}\right)-(1 \\
& \left.+\frac{4}{3} \sin 1\right) x_{2}+\left(-2.829281750769593 \sin \left(x_{1}\right)\right. \\
& \left.+2.728194267422565 x_{2}\right) \lambda \\
& +\left(3.637592356563420 \sin \left(x_{1}\right)\right. \\
& \left.\quad-3.408364188910562 x_{2}\right) \lambda^{2} \\
& +\left(-4.544485585214082 \sin \left(x_{1}\right)\right. \\
& \left.+4.382115538190247 x_{2}\right) \lambda^{3} \\
& +\left(5.842820717586996 \sin \left(x_{1}\right)\right. \\
& \left.+5.474626880638770 x_{2}\right) \lambda^{4}+\cdots
\end{aligned}
$$

Here $m=n=1, l=m-n+1=1$ and we set $d=l$.

Step 1. Set the initial value as $v_{0}^{(0)}=1, v_{0}^{(1)}=\lambda, v_{0}^{(2)}=\lambda^{2}$, computing

$$
\begin{aligned}
& \left(c_{1}, c_{1}\right)=18.654952992399824, \\
& \left(c_{1}, c_{2}\right)=-23.623503547631628 .
\end{aligned}
$$

Step 2. Compute $v_{1}^{(0)}$ and $v_{1}^{(1)}$ by (29)

$$
\begin{aligned}
v_{1}^{(0)} & =v_{0}^{(0)}-\frac{\left(c_{1}, \phi^{(1)}\left(v_{0}^{(0)}\right)\right)}{\left(c_{1}, \phi^{(1)}\left(v_{0}^{(1)}\right)\right)} v_{0}^{(1)}=1-\frac{\left(c_{1}, c_{1}\right)}{\left(c_{1}, c_{2}\right)} \lambda \\
& =1+0.789677659572644 \lambda . \\
v_{1}^{(1)} & =v_{0}^{(1)}-\frac{\left(c_{1}, \phi^{(1)}\left(v_{0}^{(1)}\right)\right)}{\left(c_{1}, \phi^{(1)}\left(v_{0}^{(2)}\right)\right)} v_{0}^{(2)}=\lambda-\frac{\left(c_{1}, c_{2}\right)}{\left(c_{1}, c_{3}\right)} \lambda^{2} \\
& =\lambda+0.788390924640188 \lambda^{2} .
\end{aligned}
$$


Step 3. Obtain the generating polynomial of $f(x, \lambda)$ function-value Padé-type approximant by (27)

$$
\begin{aligned}
v_{2}^{(0)} & =v_{1}^{(0)}-\frac{\left(c_{1}, \phi^{(2)}\left(v_{1}^{(0)}\right)\right)}{\left(c_{1}, \phi^{(2)}\left(v_{1}^{(1)}\right)\right)} v_{1}^{(1)} \\
& =1+1.1752 \times 10^{-13} \lambda-0.622574700198084 \lambda^{2}
\end{aligned}
$$

Step 4. From (10) get the denominator of $f(x, \lambda)$ functionvalue Padé-type approximant

$$
\begin{aligned}
\tilde{v}_{2}(\lambda) & =\lambda^{2} v_{2}\left(\lambda^{-1}\right)=b_{0} \lambda^{2}+b_{1} \lambda+b_{2} \\
& =-1.606232954345608 \lambda^{2}-1.89 \times 10^{-13} \lambda+1
\end{aligned}
$$

Step 5. Get the numerator of $f(x, \lambda)$ function-value Padétype approximant by (12)

$$
\begin{aligned}
\widetilde{w}_{2}(x, \lambda)= & \left(b_{0} c_{0}+b_{1} c_{1}+b_{2} c_{2}\right) \lambda^{2}+\left(b_{1} c_{0}+b_{2} c_{1}\right) \lambda \\
& +b_{2} c_{0}=a_{2} \lambda^{2}+a_{1} \lambda+a_{0}
\end{aligned}
$$

where

$$
\begin{aligned}
a_{2}= & 1.495948417436477 \sin x_{1}-9.0779 \times 10^{-16} x_{2} \\
& -1.606232954345608 x_{1} \cos x_{2} \\
a_{1}= & -2.829281750769845 \sin x_{1} \\
& +2.728194267422966 x_{2}-1.89 \\
& \times 10^{-13} x_{1} \cos x_{2} \\
a_{0}= & 1.3333333333333333 \sin x_{1} \\
& -2.121961313077195 x_{2}+x_{1} \cos x_{2}
\end{aligned}
$$

Step 6. Obtain $f(x, \lambda)$ function-valued Padé-type approximant

$$
\left(\frac{2}{2}\right)_{f}(x, \lambda)=\frac{a_{2} \lambda^{2}+a_{1} \lambda+a_{0}}{b_{0} \lambda^{2}+b_{1} \lambda+b_{2}} .
$$

\section{Numerical Results}

In this section, we will consider the square domain case of $D=[\alpha, \beta] \times[\alpha, \beta]$. We apply Algorithm 7 for 2DFPTA to approximate the corresponding 2DF-II [11]. Compared with the methods in $[4,18]$, numerical experiments show that our method improves the calculation accuracy. Furthermore, our method can estimate the corresponding characteristic values of $f(x, \lambda)$ from the real roots of $\widetilde{v}_{n}(\lambda)$.

Example 9. Consider the same Fredholm integral equation with Example $8[4,18]$.

When $\lambda=1$, the exact solution of (43) is

$$
f\left(x_{1}, x_{2}, \lambda\right)=x_{1} \cos \left(x_{2}\right)-x_{2} \text {. }
$$

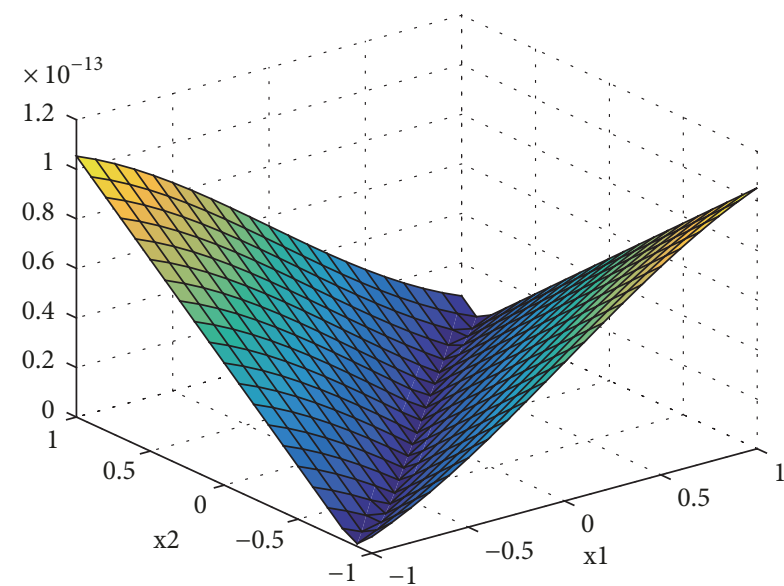

Figure 1: $\lambda=1$, error of $(2 / 2)_{f}(x, \lambda)$ in Example 9.

By Algorithm 7, we get the following calculation results:

$$
\begin{aligned}
\widetilde{v}_{2}(\lambda)= & -1.89 \times 10^{-13} \lambda \\
& -1.606232954345608 \lambda^{2}, \\
\left(\frac{2}{2}\right)_{f}\left(x_{1}, x_{2}, 1\right)= & -x_{2}+x_{1} \cos \left(x_{2}\right)+5.8593 \\
& \times 10^{-14} \sin \left(x_{1}\right)+5.6 \\
& \times 10^{-14} x_{2},
\end{aligned}
$$

where when $\lambda=1$ the maximum absolute error of (2/ $2)_{f}\left(x_{1}, x_{2}, 1\right)$ caused by Algorithm 7 is shown in Figure 1. From Figure 1, we can observe that the error is $1.05 \times 10^{-13}$ and in the process we just only employ the first $m+n=4$ items of Neumann series (4), while in [4] the error was $8.7 \times 10^{-5}$ when the first 5 items of Chebyshev series corresponding to this equation were used and in [18] the corresponding error was $5.3 \times 10^{-13}$.

By Algorithm 7, we also get that

$$
\begin{aligned}
& \tilde{v}_{3}(\lambda)=+0.999999999999785 \lambda \\
&-1.606232954345953 \lambda^{2} \\
&-1.606232954345763 \lambda^{3} \\
& \widetilde{v}_{4}(\lambda)=1-7.837686719857387 \lambda \\
&-2.094833520416517 \lambda^{2} \\
&+12.589150695272894 \lambda^{3} \\
&+0.784806330738950 \lambda^{4}
\end{aligned}
$$

where their eigenvalue has estimates; see Table 1.

In the computational process of $\widetilde{v_{2}}(\lambda)$, we get two possible characteristic values $\lambda_{1}=0.7890340298098$ and $\lambda_{2}=-0.789034029809980$. After further computing we can 
TABLE 1: Estimates of the characteristic values of $(n / n)_{f}(x, \lambda), n=1,2,3,4$ in Example 9.

\begin{tabular}{lcccc}
\hline$(n / n)_{f}$ & $(1 / 1)_{f}$ & $(2 / 2)_{f}$ & $(3 / 3)_{f}$ & $(4 / 4)_{f}$ \\
\hline$\lambda_{1}$ & 0.789677659572644 & 0.789034029809863 & 0.789034029809820 & 0.789034029809775 \\
$\lambda_{2}$ & & -0.789034029809980 & -0.789034029809980 & -0.789034029809980 \\
$\lambda_{3}$ & & -0.999999999999958 & -16.167681854028405 \\
$\lambda_{4}$ & & & & 0.126589673465619 \\
\hline
\end{tabular}

TABLE 2: When $\lambda=1$, the errors of $(2 / 2)_{f}(x, \lambda)$ and $(2 / 2)_{u}(x, \lambda)$ at corresponding points in Example 9.

\begin{tabular}{lccc}
\hline$x$ & $f(x, \lambda)$ & $\left|f(x, \lambda)-(2 / 2)_{u}(x, \lambda)\right|$ & $f(x, \lambda)-(2 / 2)_{f}(x, \lambda) \mid$ \\
\hline$(-0.1,0.1)$ & $-1.9950 \mathrm{e}-001$ & $2.9438 \mathrm{e}-014$ & $2.4951 \mathrm{e}-016$ \\
$(-0.2,0.2)$ & $-3.9601 \mathrm{e}-001$ & $5.8583 \mathrm{e}-014$ & $4.4057 \mathrm{e}-016$ \\
$(-0.3,0.3)$ & $-5.8660 \mathrm{e}-001$ & $8.7142 \mathrm{e}-014$ & $5.1532 \mathrm{e}-016$ \\
$(-0.4,0.4)$ & $-7.6842 \mathrm{e}-001$ & $1.1483 \mathrm{e}-013$ & $4.1707 \mathrm{e}-016$ \\
$(-0.5,0.5)$ & $-9.3879 \mathrm{e}-001$ & $1.4137 \mathrm{e}-013$ & $9.0830 \mathrm{e}-017$ \\
$(0.6,-0.6)$ & $1.0952 \mathrm{e}+000$ & $1.6650 \mathrm{e}-013$ & $5.1608 \mathrm{e}-016$ \\
$(0.7,-0.7)$ & $1.2354 \mathrm{e}+000$ & $1.8996 \mathrm{e}-013$ & $1.4536 \mathrm{e}-015$ \\
$(0.8,-0.8)$ & $1.3574 \mathrm{e}+000$ & $2.1153 \mathrm{e}-013$ & $2.7682 \mathrm{e}-015$ \\
$(0.9,-0.9)$ & $1.4594 \mathrm{e}+000$ & $2.3098 \mathrm{e}-013$ & $4.5028 \mathrm{e}-015$ \\
$(1.0,-1.0)$ & $1.5403 \mathrm{e}+000$ & $2.4813 \mathrm{e}-013$ & $6.6960 \mathrm{e}-015$ \\
\hline
\end{tabular}

deduce that $\widetilde{v}_{3}(\lambda)$ and $\widetilde{v}_{4}(\lambda)$ both include the two real roots. Therefore we consider the two numbers as characteristic values of this equation.

In Table 2, $(2 / 2)_{f}(x, \lambda)$ is from Algorithm 7 in the paper and $(2 / 2)_{u}(x, \lambda)$ is from the method in [18], where

$$
\begin{aligned}
\left(\frac{2}{2}\right)_{u}\left(x_{1}, x_{2}, 1\right)= & -x_{2}+x_{1} \cos \left(x_{2}\right)+2.94875 \\
& \times 10^{-13} \sin \left(x_{1}\right) .
\end{aligned}
$$

We find that from the numerical results $(2 / 2)_{f}(x, \lambda)$ has better approximation effect compared with $(2 / 2)_{\mathcal{u}}(x, \lambda)$ in [18].

Example 10. Consider the following [4, 18]:

$$
\begin{aligned}
& f\left(x_{1}, x_{2}, \lambda\right) \\
& =x_{1} e^{-x_{2}}+\left(\frac{1}{3} e^{-1}-\frac{7}{12}\right) x_{1}-\frac{1}{2} x_{2} \\
& +\lambda \int_{0}^{1} \int_{0}^{1}\left(x_{1} y_{1}+x_{2} e^{y_{2}}\right) f\left(y_{1}, y_{2}, \lambda\right) d y_{1} d y_{2}, \\
& x_{1}, x_{2} \in[0,1] .
\end{aligned}
$$

When $\lambda=1$, the exact solution of (56) is

$$
f\left(x_{1}, x_{2}, \lambda\right)=x_{1} e^{-x_{2}}+x_{2} .
$$

By Algorithm 7, the calculation of Example 10 is shown as follows:

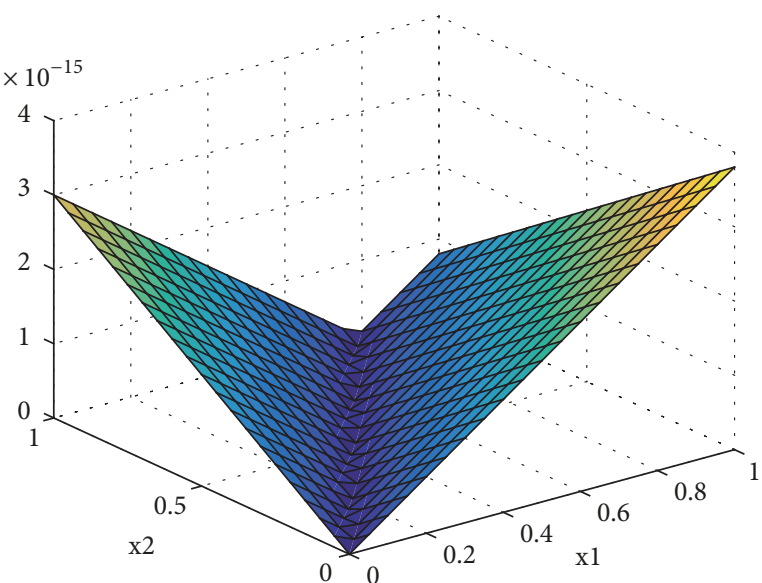

Figure 2: $\lambda=1$, error of $(2 / 2)_{f}(x, \lambda)$ in Example 10.

$$
\begin{aligned}
\widetilde{v}_{2}(\lambda)= & -1.333333333334607 \lambda \\
& +0.118548104777520 \lambda^{2}, \\
\left(\frac{2}{2}\right)_{f}\left(x_{1}, x_{2}, 1\right)= & -3.801972000573112 \times 10^{-15} x_{1} \\
& +1.000000000000003 x_{2} \\
& +x_{1} e^{-x_{2}},
\end{aligned}
$$

where when $\lambda=1$ the maximum absolute error of $(2 / 2)_{f}\left(x_{1}, x_{2}, 1\right)$ caused by Algorithm 7 is shown in Figure 2. From Figure 2, we can observe that the error is $3.7 \times 10^{-15}$ and in the process we just only employ the first $m+n=4$ items of Neumann series (4). While in [4] the error was $8.8 \times 10^{-7}$ 
TABLE 3: Estimates of the characteristic values of $(n / n)_{f}(x, \lambda), n=1,2,3,4$ in Example 10.

\begin{tabular}{|c|c|c|c|c|}
\hline$(n / n)_{f}$ & $(1 / 1)_{f}$ & $(2 / 2)_{f}$ & $(3 / 3)_{f}$ & $(4 / 4)_{f}$ \\
\hline$\lambda_{1}$ & 0.815393639551284 & 0.808054702319182 & 0.808054702319182 & 0.808054702319181 \\
\hline$\lambda_{2}$ & & 10.439137615545988 & 10.439137615545846 & 10.439137615544714 \\
\hline$\lambda_{3}$ & & & 0.017857142857281 & $-0.0476584+0.4291069 i$ \\
\hline$\lambda_{4}$ & & & & $-0.0476584-0.4291069 \mathrm{i}$ \\
\hline
\end{tabular}

TABLE 4: When $\lambda=1$, the errors of $(2 / 2)_{f}(x, \lambda)$ and $(2 / 2)_{\mathcal{u}}(x, \lambda)$ at corresponding points in Example 10.

\begin{tabular}{lccc}
\hline$x$ & $f(x, \lambda)$ & $\left|f(x, \lambda)-(2 / 2)_{u}(x, \lambda)\right|$ & $f(x, \lambda)-(2 / 2)_{f}(x, \lambda) \mid$ \\
\hline$(0.1,0.1)$ & $1.9048 \mathrm{e}-001$ & $9.9048 \mathrm{e}-016$ & $8.0197 \mathrm{e}-017$ \\
$(0.2,0.2)$ & $3.6375 \mathrm{e}-001$ & $1.9637 \mathrm{e}-015$ & $1.6039 \mathrm{e}-016$ \\
$(0.3,0.3)$ & $5.2225 \mathrm{e}-001$ & $2.9222 \mathrm{e}-015$ & $2.4059 \mathrm{e}-016$ \\
$(0.4,0.4)$ & $6.6813 \mathrm{e}-001$ & $3.8681 \mathrm{e}-015$ & $3.2079 \mathrm{e}-016$ \\
$(0.5,0.5)$ & $8.0327 \mathrm{e}-001$ & $4.8033 \mathrm{e}-015$ & $4.0099 \mathrm{e}-016$ \\
$(0.6,0.6)$ & $9.2929 \mathrm{e}-001$ & $5.7293 \mathrm{e}-015$ & $4.8118 \mathrm{e}-016$ \\
$(0.7,0.7)$ & $1.0476 \mathrm{e}+000$ & $6.6476 \mathrm{e}-015$ & $5.6138 \mathrm{e}-016$ \\
$(0.8,0.8)$ & $1.1595 \mathrm{e}+000$ & $7.5595 \mathrm{e}-015$ & $6.4158 \mathrm{e}-016$ \\
$(0.9,0.9)$ & $1.2659 \mathrm{e}+000$ & $8.4659 \mathrm{e}-015$ & $7.2177 \mathrm{e}-016$ \\
$(1.0,1.0)$ & $1.3679 \mathrm{e}+000$ & $9.3679 \mathrm{e}-015$ & $8.0197 \mathrm{e}-016$ \\
\hline
\end{tabular}

when the first 5 items of Chebyshev series corresponding to this equation were used and in [18] the corresponding error was $5.5 \times 10^{-15}$.

From $\widetilde{v_{2}}(\lambda)$ in (58), we get two possible characteristic values $\lambda_{1}=0.80805470231918, \lambda_{2}=10.43913761554$. By Algorithm 7, we also get that

$$
\begin{aligned}
& \tilde{v}_{3}(\lambda)=-0.428436108131838 \lambda \\
&-1.087981528825263 \lambda^{2} \\
&+0.107273851063696 \lambda^{3}, \\
& \widetilde{v}_{4}(\lambda)=1-1.225739535044527 \lambda \\
&-0.111597656991711 \lambda^{2} \\
&+0.128338192937178 \lambda^{3} \\
&-0.010276622714483 \lambda^{4} .
\end{aligned}
$$

Note that both $\widetilde{v}_{3}(\lambda)$ and $\widetilde{v}_{4}(\lambda)$ include the two real roots. Therefore, we consider the two real roots as the characteristic values of (56). For estimates of the characteristic values of $(n / n)_{f}(x, \lambda), n=1,2,3,4$ in Example 10, see Table 3.

In Table $4,(2 / 2)_{f}(x, \lambda)$ is from Algorithm 7 in the paper and $(2 / 2)_{u}(x, \lambda)$ is from the method in [18], where

$$
\begin{aligned}
\left(\frac{2}{2}\right)_{u}\left(x_{1}, x_{2}, 1\right)= & 1.000000000000009 x_{2} \\
& +1.000000000000001 x_{1} e^{-x_{2}} .
\end{aligned}
$$

From the numerical results in Table $4,(2 / 2)_{f}(x, \lambda)$ has better approximation effect compared with $(2 / 2)_{u}(x, \lambda)$ in [18].

\section{Data Availability}

The [tables] data used to support the findings of this study are included within the article. The [figures] data used to support the findings of this study are included within the article. The [software codes] data used to support the findings of this study are available from the corresponding author upon request.

\section{Conflicts of Interest}

The authors declare that they have no conflicts of interest.

\section{Acknowledgments}

This work is funded by the National Natural Science Foundation of China under Grant no. 11371243, Key Disciplines of Shanghai Municipality under Grant no. S30104, the Fostering Master's Degree Empowerment Point Project of Hefei University under Grant no. 2018xs03, and the Key Natural Science Research Project of University of Anhui Province, Education Department of Anhui Province, under Grant nos. KJ2017A547, KJ2016A592, and KJ2018A565.

\section{References}

[1] A. Kadem and D. Baleanu, "Two-dimensional transport equation as Fredholm integral equation," Communications in Nonlinear Science and Numerical Simulation, vol. 17, no. 2, pp. 530-535, 2012.

[2] S. Hatamzadeh-Varmazyar and Z. Masouri, "Numerical method for analysis of one- and two-dimensional electromagnetic scattering based on using linear Fredholm integral equation models," Mathematical and Computer Modelling, vol. 54, no. 910, pp. 2199-2210, 2011. 
[3] K. Atkinson, D. D. Chien, and J. Seol, "Numerical analysis of the radiosity equation using the collocation method," Electronic Transactions on Numerical Analysis, vol. 11, pp. 94-120, 2000.

[4] Z. Avazzadeh and M. Heydari, "Chebyshev polynomials for solving two dimensional linear and nonlinear integral equations of the second kind," Computational \& Applied Mathematics, vol. 31, no. 1, pp. 127-142, 2012.

[5] G. Han and R. Wang, "Richardson extrapolation of iterated discrete Galerkin solution for two-dimensional Fredholm integral equations," Journal of Computational and Applied Mathematics, vol. 139, no. 1, pp. 49-63, 2002.

[6] G. Karkarashvili, "On a numerical solution of the twodimensional Fredholm integral equation of the second kind," A. Razmadze Mathematical Institute of the Georgian Academy of Sciences., vol. 139, pp. 33-44, 2005.

[7] F. Liang and F.-R. Lin, "A fast numerical solution method for two dimensional Fredholm integral equations of the second kind based on piecewise polynomial interpolation," Applied Mathematics and Computation, vol. 216, no. 10, pp. 3073-3088, 2010.

[8] D. Occorsio and M. G. Russo, "Numerical methods for Fredholm integral equations on the square," Applied Mathematics and Computation, vol. 218, no. 5, pp. 2318-2333, 2011.

[9] A. Tari and S. Shahmorad, "A computational method for solving two-dimensional linear Fredholm integral equations of the second kind," The ANZIAM Journal, vol. 49, no. 4, pp. 543-549, 2008.

[10] W.-J. Xie and F.-R. Lin, "A fast numerical solution method for two dimensional Fredholm integral equations of the second kind," Applied Numerical Mathematics, vol. 59, no. 7, pp. 17091719, 2009.

[11] Q. Wang and H. Wang, "Meshless method and convergence analysis for 2-dimensional Fredholm integral equation with complex factors," Journal of Computational and Applied Mathematics, vol. 304, pp. 18-25, 2016.

[12] Jin Xie, "A Geometric Modeling Method Based on TH-Type Uniform B-Splines," Mathematical Problems in Engineering, vol. 2014, Article ID 242469, 7 pages, 2014.

[13] P. R. Graves-Morris, "Solution of integral equation using generalized inverse function-valued Padé Approximants, I," Journal of Computational and Applied Mathematics, vol. 32, no. 1-2, pp. 117-124, 1990.

[14] C. J. Li and C. Q. Gu, "Epsilon-algorithm and eta-algorithm of generalized inverse function-valued Padé approximants using for solution of integral equations," Applied Mathematics and Mechanics. Yingyong Shuxue He Lixue, vol. 24, no. 2, pp. 197204, 2003.

[15] C. Q. Gu, B. Z. Pan, and B. B. Wu, "Orthogonal polynomials and determinant formulas of function-valued Padé -type approximation using for solution of integral equations," Applied Mathematics and Mechanics. Yingyong Shuxue He Lixue, vol. 27, no. 6, pp. 750-756, 2006.

[16] C. Gu and J. Shen, "Function-valued Padé -type approximant via the formal orthogonal polynomials and its applications in solving integral equations," Journal of Computational and Applied Mathematics, vol. 221, no. 1, pp. 114-131, 2008.

[17] C. Gu and Y. Tao, "Function-valued Padé -type approximant via Padé -algorithm and its applications in solving integral equations," Applied Mathematics and Computation, vol. 217, no. 20, pp. 7975-7984, 2011.
[18] Y. Tao and D. Wang, "Padé -type approximation method for two dimensional Fredholm integral equations of the second kind," Applied Mathematics and Computation, vol. 246, pp. 71-78, 2014.

[19] C. Brezinski, Computational aspects of linear control, vol. 1 of Numerical Methods and Algorithms, Kluwer Academic Publishers, Dordrecht, 2002.

[20] P. R. Graves-Morris, "Padé approximants for linear integral equations?" Journal of the Institute of Mathematics and Its Applications, vol. 21, no. 4, pp. 375-378, 1978. 


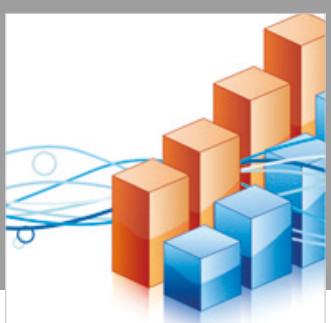

Advances in

Operations Research

\section{-n-m}
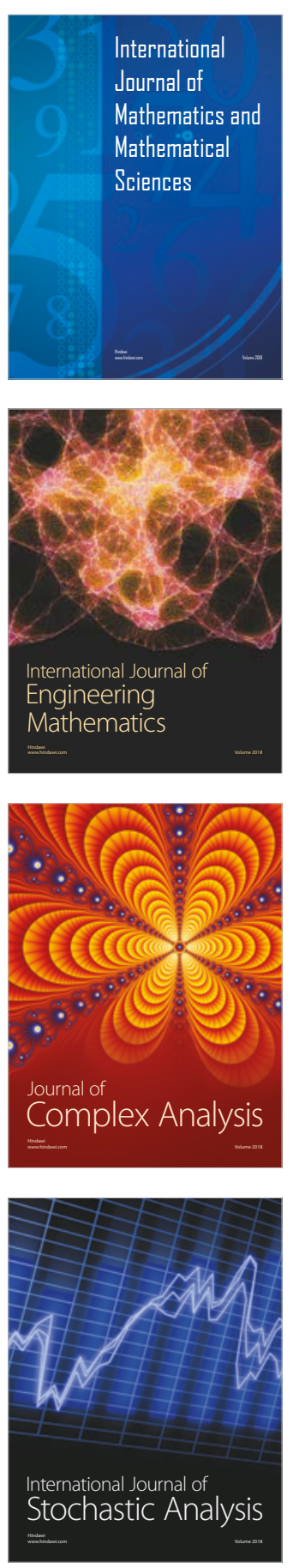
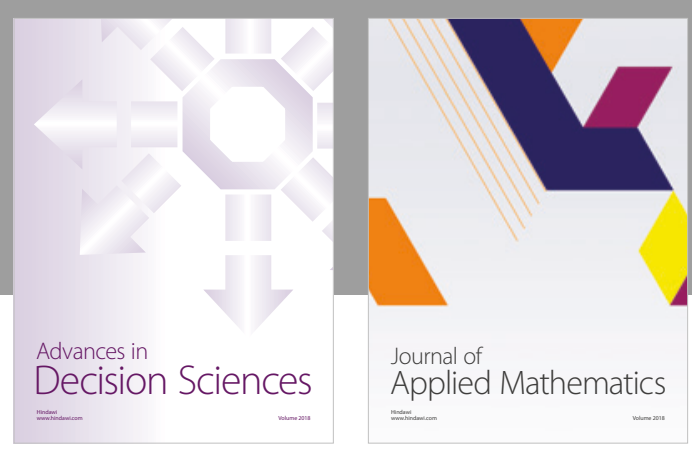

Journal of

Applied Mathematics
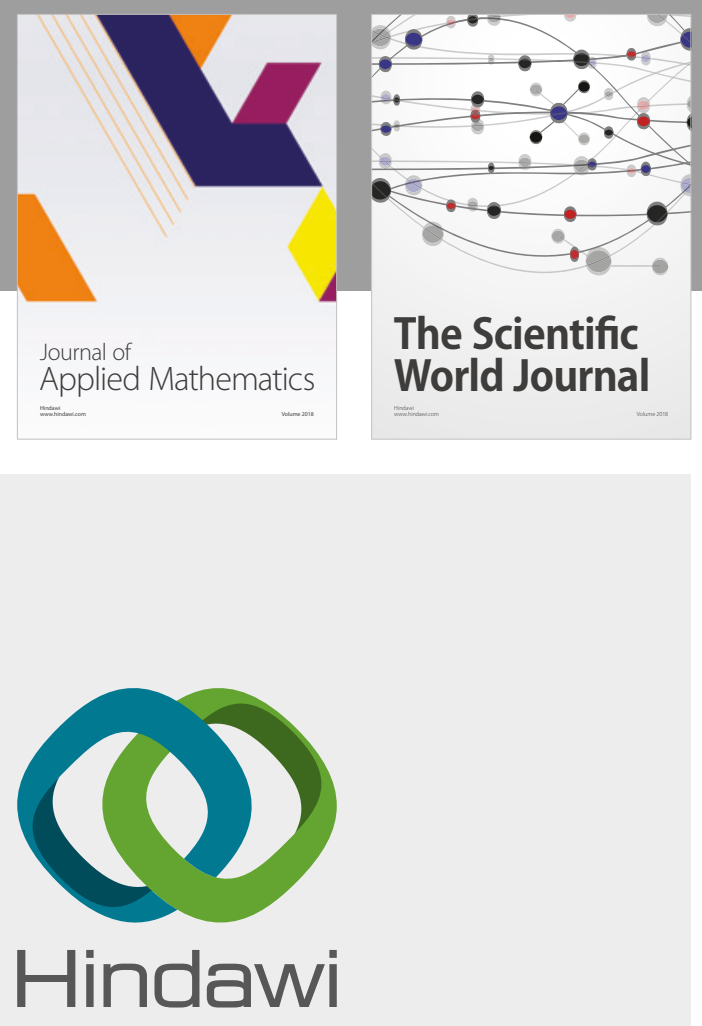

Submit your manuscripts at

www.hindawi.com

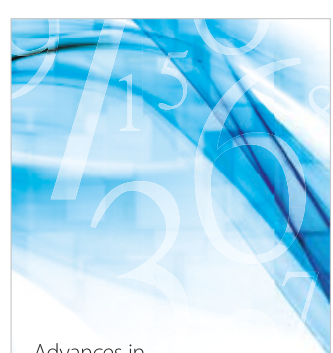

Advances in
Numerical Analysis
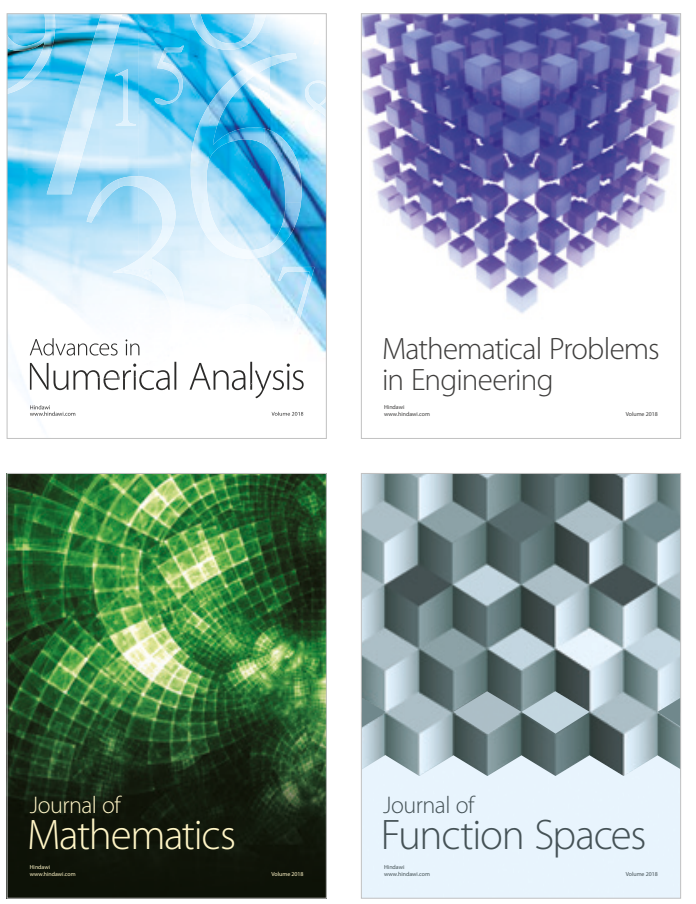

Mathematical Problems in Engineering

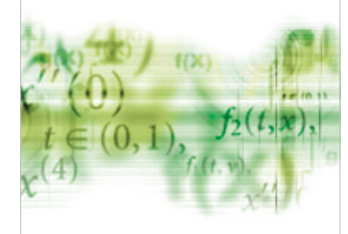

International Journal of

Differential Equations

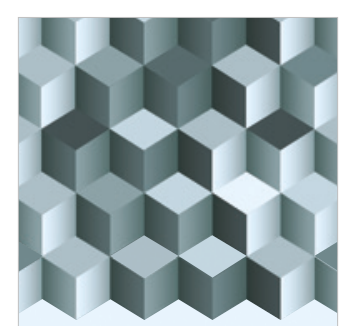

Journal of

Function Spaces

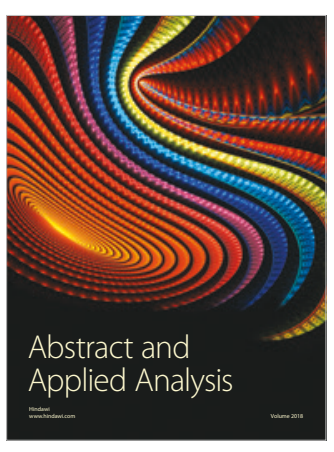

The Scientific

World Journal

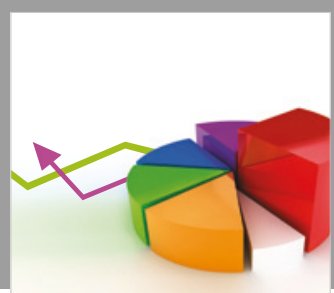

Journal of

Probability and Statistics
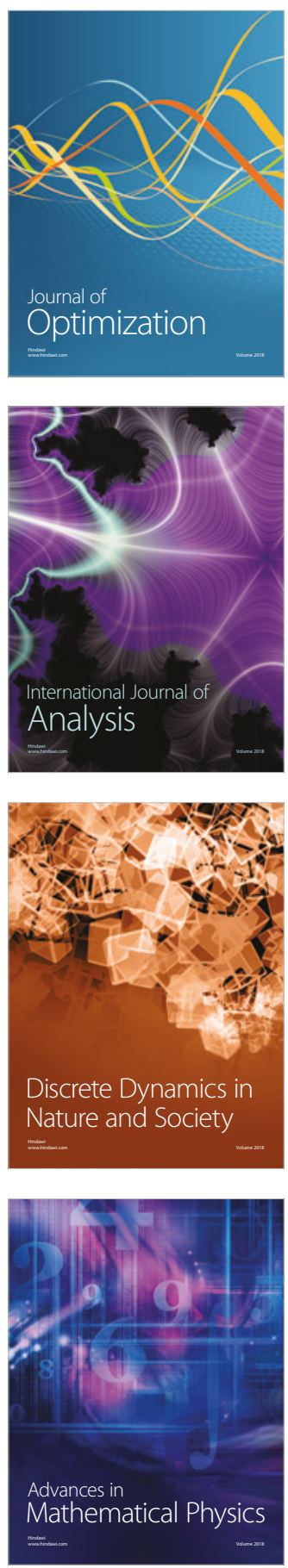